\title{
Full model wavenumber inversion (FMWI): identifying sources of information for the elusive middle model wavenumbers
}

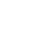

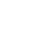
3

\author{
Tariq Alkhalifah[1], BingBing Sun[1], and Zedong Wu[1] \\ [1]Physical Sciences and Engineering \\ King Abdullah University of Science and Technology \\ Mail box \# 1280 \\ Thuwal 23955-6900 \\ Saudi Arabia \\ (July 9, 2018) \\ Running head: Middle model wavenumbers?
}

\begin{abstract}
We recognized over the years that our conventional surface seismic recording can effectively identify two main features of the Earth: its seismic propagation attributes and those attributes resulting in echoes or reflections. Thus, the resulting expression of the Earth is dominated by the generally smooth (long wavelength) features that control wave propagation, which we use to invert for the short wavelength features causing reflections in a process we refer to as migration velocity analysis (MVA) and imaging, respectively. The features of the Earth that fall in between these two model scales: the middle wavenumbers, have been elusive, which is a dilemma for full waveform inversion (FWI) as we need to build the full velocity model (a broad band of model wavenumbers). We analyse the middle model wavenumber gap, but focus more here on potential sources of information for
\end{abstract}


such middle model wavenumbers. Such sources include regularization, objective function enhancements, and multi scattered energy. Regularization, provided by a total variation constraint admits middle and high model wavenumbers components into the model to enforce the model's compliance with such a constraint. As opposed to minimizing the total variation, such a constraint merely reduces the model space, and thus, these injected middle model wavenumbers are as good as the projected data information to the reduced model space. Such data information includes large offsets, but also multi scattered energy, in which the energy through wave path and scattering updates can admit more of the elusive middle wavenumber information that comes from the data. The combination of the right level of allowable model variations with the added data information from large offsets and multi scattering can help in filling the elusive middle model wavenumber gap and admit plausible models. 


\section{INTRODUCTION}

The recorded pulses of seismic waves originating from an active source and penetrating the Earth include generally information related to the average velocity of the propagating wave, manifested in the traveltime it took for the pulse to arrive, but more importantly, possibly some information of where it bounced from, usually referred to as velocity contrasts (Aki and Richards, 1980). The bounce location information (imaging) requires more than one pulse measurement from the same bouncing interface, providing directional information (Yilmaz, 2001). The traveltime and bounce roles were highlighted in Claerbout (1985), where he attempts to describe the data sensitivity to the various velocity model scales.

The objective of a full waveform inversion (FWI) implementation is to predict the velocity information within a domain of interest that is missing from the initial (known) model of the subsurface. The missing velocity information is iteratively added to the known model until the resulting combined model is able to produce simulated data that resembles the recorded data. In doing so, FWI uses the difference between the recorded data and the simulated ones to update the velocity model by mapping this difference to updates in the model space (Tarantola, 1984). These updates are extracted from wavefields, and thus, they are sinusoidal in nature best expressed by wavenumber components in the space domain (Wu and Toksaz, 1987; Sirgue and Pratt, 2004; Alkhalifah, 2016a). So to predict the missing velocity information required to produce the desired simulated data, we will need FWI to find all the wavenumber components that could properly represent such missing information. The initial model often contains only smooth components, low wavenumber information. So FWI needs to inject wavenumbers into the model from low to high as we have to satisfy the propagation accuracy requirement for various frequencies to avoid cycle 
skipping (Bunks et al., 1995; Pratt et al., 1996; Alkhalifah, 2014).

The middle wavenumber gap is dependent on our recording geometry, velocity model, and the recorded frequency band (Wu and Toksaz, 1987). For surface seismic data, the gap often widens with depth due to the limited illumination angles. Deeper parts of the model also suffer from natural physical limitations of wave propagation like energy loss due to geometrical spreading and dispersion, which also attenuates the high frequency content. The dilemma in resolving the deep model portion from surface seismic data is an ongoing challenge. Attempts to fill the middle wavenumber gap sparked the push for more low-frequency acquisition (Bunks et al., 1995; Pratt et al., 1996; Virieux and Operto, 2009) and for acquiring larger offsets (Sirgue and Pratt, 2004), as well as, improving our ability to estimate a good initial model for FWI (Xu et al., 2012; Ma et al., 2012; Fleury and Perrone, 2012; Wang et al., 2013; Alkhalifah and Wu, 2016b; Chavent and Plessix, 1999; Clement et al., 2001).

In a previous paper, Alkhalifah (2016a) demonstrated the importance of accessing the middle model wavenumbers and the necessity to build such model wavenumbers from low to high. He emphasizes the case for using scattering-angle filtering to implement this wavenumber continuation from low to high. However, the sources for accessing such missing middle model wavenumbers from the data were not addressed. In this paper, we will investigate combining two, one of them often ignored, sources of middle wavenumber information. We will show the potential of these sources of model wavenumber information, but also outline their limitations. Most importantly, we seek to gain knowledge on how to use such information in waveform inversion. We will start the paper by reviewing FWI from the model wavenumber standpoint, and then shift to the issue of the middle wavenumber gap. Finally, we investigate the role of regularization and multi scattering in providing 
such middle model wavenumbers. We will support the theoretical assertions with numerical examples.

\section{FULL WAVEFORM INVERSION IN A NUTSHELL}

The difference between the recorded field data and data simulated in our computing devices, using the same experiment setup, and under our best assumptions of the physics of wave propagation inside the Earth, is used to update the Earth's acoustic, elastic, and possibly anisotropic model of the Earth. This update is often implemented in an iterative framework we refer to as full waveform inversion (FWI). So we repeatedly map the data difference (between field and simulated data, we refer to as residual) to updates in the model using a linearized approximation of an otherwise nonlinear relation between model and data. If such difference between the field and simulated data decreases over iterations, we are converging. However, we often ask ourselves, what are we converging to? We often desire the minimum difference (global minimum) and here we will offer approaches to help do so. The global minimum ability to represent the true velocity model depends on the accuracy of our physical representation of the Earth (simulation) and our ability to illuminate the model space, or impose the proper constraints. The measure of difference can be performed in many ways, the most popular is given by a simple point-by-point subtraction adhering to the least square $l_{2}$ norm objective function first suggested within the framework of FWI by Tarantola (1984)

$$
E_{F W I}(m)=\sum_{i}\left|d_{o_{i}}-d_{s_{i}}(m)\right|^{2}
$$

We define $i$ as the source index, $d_{o_{i}}$ as the observed data, $d_{s_{i}}=u_{s}\left(\mathbf{x}_{s_{i}}, t\right)$ as the modeled synthetic data measured at the surface, $z=0$, and $m(\mathbf{x})$ as (any form of) the velocity 
model described in space using the vector $\mathbf{x}=\{x, y, z\}$, along the Cartesian coordinates, with $t$ as time. From this point on, and for simplicity, but without the loss of generality, we represent wavefields and data in the frequency domain. In this case, the modeled wavefield, $u_{s}$, satisfies the following wave equation:

$$
L(m) u_{s_{i}}=f(\omega) \delta\left(\mathbf{x}-\mathbf{x}_{s_{i}}\right)
$$

for a particular source location, $\mathbf{x}_{s_{i}}$, and $f$ is the source function given in the frequency domain, with

$$
L(m)=L^{*}(m)=\nabla^{2}+\omega^{2} m(\mathbf{x}),
$$

for the case of an acoustic isotropic constant-density Earth, where $\omega$ is the angular frequency and $\nabla^{2}$ is the Laplacian operator $(=\nabla \cdot \nabla$, where $\nabla$ is the gradient). In this case, $m(\mathbf{x})$ is given by the slowness squared. However, the Helmholtz operator, $L$, can have differing forms to represent more complex physics, but for the purpose of focusing on model wavenumbers, we will stick with the simplest form, which happens to render the operator self adjoint. The gradient corresponding to such an objective function is given by (Tarantola, 1984)

$$
R_{F W I}(\mathbf{x})=\Re\left\{\frac{\partial L}{\partial m} \sum_{i} u_{s_{i}}(\mathbf{x}) L^{-1} \sum_{j} \delta\left(\mathbf{x}-\mathbf{x}_{r_{i j}}\right)\left(d_{o_{i j}}-d_{s_{i j}}\right)^{*}\right\}
$$

where $\mathbf{x}_{r_{i j}}$ are the locations of the receivers for source $i$, and $j$ is the receiver index. The gradient (as a vector) points to the objective functional steepest descent direction in the model space. An often used simple update of the model, is to combine this steepest descent vector with a line search for a minimum.

Such Born approximation based gradients, which correspond to the interaction of a 
source wavefield with the adjoint residual wavefield, are space domain functions with a harmonic nature courtesy of the wavefields used in computing them. These harmonic features, using a local plane wave decomposition of the wavefields, can be expressed as a function of a model amplitude (radiation pattern), and a model wavenumber vector. The model wavenumber vector describes the resolution and dip of a potential gradient point (estimated model perturbation). Based on diffraction tomography, and assuming locally plane wavefields for the state and adjoint state wavefields, the model wavenumber vector, $\mathbf{k}_{\mathbf{m}}$, is given by (Miller et al., 1987; Jin et al., 1992; Thierry et al., 1999),

$$
\mathbf{k}_{\mathbf{m}}=\mathbf{k}_{\mathbf{s}}+\mathbf{k}_{\mathbf{r}}=2 \frac{\omega}{v} \cos \frac{\theta}{2} \mathbf{n}
$$

which depends on, among other things, the angular frequency, $\omega$, with a direction guided by a unit vector, $\mathbf{n}$, normal to a potential scattering interface. Here, $\mathbf{k}_{\mathbf{s}}$ and $\mathbf{k}_{\mathbf{r}}$ are the source and receiver (or any state and adjoint state) wavefield wavenumber vectors, respectively, at the model point, with $\theta$ as the angle between these vectors (the scattering angle), and $v$ $\left(=\frac{1}{\sqrt{m}}\right)$ is the velocity at the model point. For multi scattered energy, the state and adjoint state could include perturbed wavefields. Nevertheless, equation 5 still applies under the local plane wave approximation.

\section{THE MIDDLE WAVENUMBER GAP}

The updates we expect from FWI consists of, as Mora (1988) so eloquently described, transmission and scattering parts. The transmission part is provided by equation 4 when the scattering angle between the state and adjoint state wavefields is around $180^{\circ}$. It is dependent on the dominant wavelength along the wavepath, and provided by the first Fresnel zone 
(Williamson, 1991). Such updates, according to diffraction tomography (equation 5) provide low wavenumber (long wavelength) wavepath updates. For deeper targets, such transmission updates are also provided by wavepath updates along reflection paths. For transmissions in all cases, equation 5 is combined with the first fresnel zone limit (Williamson, 1991) to provide the resolution range for transmissions, and that range starts at zero along the ray path. The upper bound for transmissions can be extracted, for a homogeneous background, from simple trigonometric relations, and thus, is given by (Alkhalifah, 2016b):

$$
k_{\max }=2 k \frac{\sqrt{1+4 l k}}{1+2 l k},
$$

where $k=\frac{\omega}{v}, l$ is the length of the wavepath, for example from the source to the receiver. Note that for zero offset $(l=0)$, equation $(6)$ provides the same resolution as equation (5) for $\theta=0$. For large $l, k_{\max } \approx 2 \sqrt{\frac{k}{l}}$, which implies, as expected, resolution is inversely proportional to wavepath length for transmissions. In a homogeneous background, formula 6 applies to reflections from plane interfaces, where the $l$ corresponds to the path from the source to the reflector and back to the receiver. If the energy corresponds to scattering from a point scatterer, then $l$ corresponds to the wavepath from the point scatterer (secondary source) to the source or receiver. This path from a scatterer is far shorter, and thus, according to equation 6 , provides higher resolution. This phenomenon will be described later in multi scattering.

So the model wavenumbers we may expect at a point in the subsurface from far field transmission data (i.e. diving waves or reflection wave paths) range from zero to $2 \sqrt{\frac{k}{l}}$. For reflections, the corresponding model wavenumbers range from $2 k \cos \frac{\theta_{\max }}{2}$ to $2 k$, where $\theta_{\max }$ is the maximum scattering angle, and for conventional reflection data it is tied to 
the maximum offset. So the inherent gap in the model wavenumber build up from surface seismic data is approximately proportional to $\cos \frac{\theta_{\max }}{2}-\sqrt{\frac{1}{k l}}$, granted this quantity is positive. So for deeper model points where the reflection wavepath can be an order longer than the wavelength, and the maximum scattering angle is usually small for conventional surface seismic acquisition, the gap could be wide.

In light of Gerhard Pratt's diagram that outlines the wavenumbers recovered from the discrete frequencies used in a frequency continuation strategy (Sirgue and Pratt, 2004), we show in Figure 1 such a diagram in which we include the wavenumbers extracted usually from imaging and tomographic methods. So the wavenumbers recovered (given in yellow) from reflections in FWI is governed by equation 5, which constitutes the higher wavenumbers. While diving waves and tomographic methods based on reflections provide lower wavenumbers. At depth, the maximum offset-to-depth ratio controlling the lower bound of the higher wavenumbers reduces, and the length of the wavepath, whether it corresponds to reflections or diving waves, increases causing the upper bound of the lower wavenumbers to decrease and the middle wavenumber gap to increase. In Figure 1, we also suggest that regularization and multi-scattered update energy can fill some of the gap. In fact, limited to conventional sources of information, and no constraints on the model, such middle wavenumbers are unattainable and in the next section we try to show what that means. As illustrated in Figure 1, the middle model wavenumbers do not have a fixed range; they depend on the data and the background model, but for the purpose of this study, the middle model wavenumbers correspond, by this definition, to the gap we face in conventional FWI. 


\section{Simple model test}

We consider a one-dimensional model made up of three layers, or more importantly two interfaces, in a 2D experiment. Figure 2a depicts the one dimensional depth model in blue. The peak frequency of the source wavelet is $30 \mathrm{~Hz}$, with a spectrum of frequencies ranging between $3 \mathrm{~Hz}$ and $80 \mathrm{~Hz}$, a typical band for real seismic recorded data nowadays. The source spectrum mapped to wavenumber using the velocity of the first layer is shown in Figure $2 \mathrm{~b}$ in green. The initial model, which is kinematically accurate, but smooth is shown as the black curve in Figure 2a. Figure 3 shows a part of the shot gather corresponding to the true model (left side) and part of the gather corresponding to the initial model on the (right side). Considering the 1D nature of the model, both gathers are symmetric with respect to zero offset. The direct arrivals and mainly two reflections are apparent. We use an absorbing boundary condition on all surfaces. The multiple reflection between the two layers, in which the apex should appear at $2 \mathrm{~s}$ time, is weak. For the initial velocity model, in which the reflectors are smoothed, the reflection energy, as expected, increases with offset. Such reflection energy is kinematically accurate supporting the kinematic accuracy of the initial velocity model. Thus, this velocity model should place all wavenumbers approximately in its accurate position, mitigating the FWI nonlinearity (we are within the basin of attraction of the global minima). Since we are dealing with a 1D model and noise free data, we simulate the predicted data and calculate the gradient for a single shot, and then stack the gradient laterally to get the effect of full fold coverage of the displayed profiles. Our objective here and throughout the paper is to identify what it takes to recover the difference between the initial and true model in this simple but representative experiment. Though the Earth is often more complex, we seek to analyze the inverted models in terms of wavenumber retrieval capability. For the various tests using this model, we will stop at 15 iterations of the basic 
steepest descent with Golden-section line search updates (Press et al., 1986). Though for certain approaches, like including multi scattering, the iteration might be more expensive, the purpose here is to evaluate what are we getting in terms of model wavenumbers from the various considerations. The objective function considered in this paper is the least square misfit shown in equation 1.

Using the classic single scattering FWI, Figure 2a shows the inverted model in red for a maximum offset of $1000 \mathrm{~m}$ after 15 iterations. The resulting model suffers mainly from the elusive missing middle model wavenumbers as well as missing some high wavenumbers beyond the frequency band (thus, Gibb's phenomena). This fact is reflected in Figure 2b, which shows the spectrum of the model difference between the initial and the true model (i.e., true perturbation) plotted in blue, together with the spectrum of the inverted difference (i.e., inverted perturbations) plotted in red. On the same Figure, the black curve corresponds to the model wavenumbers provided by the initial model, which are low. The green curve, which corresponds to the spectrum of the source wavelet mapped to wavenumbers, and is the same for all experiments, acts like a filter applied to the inverted model; as a result, a poor fit of the inverted model at the high end. The mismatch between the true difference and the inverted one also happens at the low end (defined here and throughout as the middle wavenumbers, which is the region between 0.02 and 0.1 of normalized model wavenumbers, see Figure $2 \mathrm{~b}$ ). As mentioned earlier, this range for the middle model wavenumber was chosen because it represents the largest misfit between the true perturbation and the inverted one using conventional FWI, or in other words, the gap.

For a larger maximum offset of $3000 \mathrm{~m}$ (shown in Figure 2c), we seem to match the middle wavenumbers better (see Figure 2d), but at the expense of the high wavenumbers. The middle wavenumber improvement is expected as the scattering angle increases with 
offset, thus, adding more lower wavenumbers. Therefore, resolution is expected to decrease, as we try to match the imperfect inverted model to larger offsets (more iterations are possibly required for more data). This behavior has an equivalency in imaging as resolution of the image decreases as we add information from larger offsets (the stretching phenomena). However, in imaging, the larger offsets are needed to enhance the signal to noise ratio. In FWI, and as an iterative process, we can control what data to include. Thus, we probably can focus the inversion on the near offsets at later iterations. This possibility is given by the fact that FWI is an iterative process and we end up adding or subtracting wavenumbers per iteration. By focusing on the short offsets at the end, we can inject higher resolution information (i.e., shorter wavelengths).

\section{SOURCES FOR MIDDLE WAVENUMBER INFORMATION}

We will look into two potential sources of middle wavenumber information at depth. One is based on assumptions we make on the model by imposing constraints on it. So middle and high wavenumbers are injected into the model by an assumption made of the model. The other is based on utilizing parts of the data, namely multi scattering, not often utilized in the update procedure. We promote such utilization in wavepath and scattering updates. An essential part of making this multi scattered energy contribute accurately is the optimization nature of its contribution. Meaning, we ensure that such updates through Born modeling fits the residuals in the data corresponding to multi scattered energy (Alkhalifah and Wu, 2016a). 


\section{Regularization}

The options for regularization are many and they usually try to conform the model to some form we expect it to comply with. In such conformation, regularization is expected to alter the wavenumber content and, in some cases, to inject middle wavenumber information. In fact, any constraints, for example, that promote reduced variation in the model will explicitly reduce the sinusoidal nature of the model. This will inherently inject model wavenumbers that are not present in the band limited nature of our wavefield to force a more white spectrum. There are a slew of methods that aim to reduce the variation in the model and enhance blockiness (Portniaguine and Zhdanov, 1999; Guitton, 2012), like the minimum gradient support (MGS) and many others. One very popular constraint, often applied on the model to capture sharp interfaces, is limiting the total variation (TV) in the model. We will focus on the TV regularization, as opposed to the other methods, as it is widely used. However, many of the conclusions we will arrive to logically applies to the other methods. TV is a measure of the variation using the following formula:

$$
J_{T V}(m)=|\sqrt{\nabla m \cdot \nabla m+\epsilon}|_{p},
$$

where $p$ defines the norm, which is one for total variation $(p=1)$, implying a simple summation over space coordinates, and $\epsilon$ is a small positive number to mitigate the singularity at zero. The operator $\nabla$, as mentioned previously, stands for the gradient. If we set $p=2$ and $\epsilon=0$, we obtain the classic Tikhnov regularization. Here, however, we focus on the TV regularization as an example. For wavefields, total variation acts as a harmonic normalizer. In other words, it tries to reduce the sinusoidal nature of the model, which induce large variations. 
The TV $(p=1)$ gradient is given by

$$
\nabla_{m} J_{T V}=\nabla \cdot \frac{\nabla m(\mathbf{x})}{\sqrt{\nabla m \cdot \nabla m+\epsilon}}=\frac{\nabla^{2} m(\mathbf{x})}{\sqrt{\nabla m \cdot \nabla m+\epsilon}}-\frac{(\nabla m \cdot \nabla m) \nabla^{2} m(\mathbf{x})}{\sqrt{\nabla m \cdot \nabla m+\epsilon}^{3}}
$$

Since for $\epsilon=0$, the gradient equals zero as the two terms become identical, the gradient is given mainly by the Laplacian of the velocity model. This operator increases the energy of the higher wavenumber components, divided by the local variation. The role of the denominator in the gradient in equation 8 , which is the local variation (amplitude of change) depends on the size of $\epsilon$. However, this local variation is key in introducing additional middle- and high-model wavenumber information. To understand its role, let consider a one dimensional velocity model with two wavenumber sinusoidals, a low wavenumber (long wavelength) component inherent in the model courtesy of the initial guess $\left(k_{1} \approx 0\right)$, and a high wavenumber (short wavelength) component introduced by the inversion gradient of a missing reflectivity $\left(k_{2}>>0\right)$. So the velocity model is given by

$$
m(z)=m_{0}+A_{1} \sin k_{1} z+A_{2} \sin k_{2} z,
$$

and the gradient of the total variation:

$\nabla_{m} J_{T V}=-\frac{\epsilon\left(A_{1} k_{1}^{2} \sin k_{1} z+A_{2} k_{2}^{2} \sin k_{2} z\right)}{\left(A_{1}^{2} k_{1}^{2} \cos ^{2} k_{1} z+A_{2}^{2} k_{2}^{2} \cos ^{2} k_{2} z+A_{1} A_{2} k_{1} k_{2}\left(\cos \left(k_{1}+k_{2}\right) z+\cos \left(k_{1}-k_{2}\right) z\right)+\epsilon\right)^{\frac{3}{2}}}$.

From the above, the key element that has changed in the sinusoidal nature of the model is the addition of the wavenumbers $k_{1}+k_{2}$ and $k_{1}-k_{2}$. The first introduces high wavenumbers, while the latter introduces middle wavenumbers to the model when the difference between $k_{1}$ and $k_{2}$ is large. The amount of wavenumber introduction decreases with an increase in 
$\epsilon$.

The total variation can be included in the objective function, as we try to minimize it, as depicted by equation 8 . We can also apply it as a constraint; that is we limit the variation in the model to some predetermined value. We will utilize this approach in the examples in this paper following the algorithm suggested by Zhu and Chan (2008). If we apply it as a constraint, we limit the model space to those models that comply with the constraint. There are many algorithms that can guarantee this compliance. Among those are algorithms that project the update to a model space that complies with a constraint like the projection onto a convex set (POCS, (Baumstein, 2013)).

We repeat the earlier (3-layers) numerical experiment, but with a total variation constraint. So we are seeking a model that fits the data and has a limited total variation. The limit on TV is extracted from the total variation of the true model, which is in this example equal to the TV of the initial model. The inverted model for the $1000 \mathrm{~m}$ offset case after 15 iterations is shown in Figure 4a in red, and has a blocky shape similar to the true model courtesy of the limited variation constraint. As a result, the spectrum of the inverted perturbation, shown in Figure $4 \mathrm{~b}$ in red, seemingly predicted the high wavenumbers slightly better, but failed to predict the middle wavenumbers well. As we use larger offsets in the inversion, specifically up to $3000 \mathrm{~m}$, we seemingly get a better fit between the true (blue) and inverted (red) curves, as shown on Figure 4c. The model perturbation spectrum shown in Figure 4d supports such an assertion, especially in correctly predicting the middle wavenumbers. There are still some difference so let us examine what happens if we include multi scattered energy in the update of FWI. 


\section{Multi scattering}

Multi scattered energy is often ignored within the context of the FWI update. Though inverted models may produce energy upon simulation that fits the multi scattered energy, the update process, which is crucial for proper convergence, often does not utilize such energy. The critical role of multi scattering as it pertains to our objective is the additional model wavenumbers it may admit. Specifically, the additional scattering will eventually offer additional information in its unique wavepath, and often scatter at additional angles. In fact, if the additional scattering is caused by an object acting as a point scatterer (smaller than a fourth of the dominant wavelength), the scattering will enhance the resolution of wavepath updates, especially if the path between the scattering object and the sensor is short. Figure 5a displays an example where such a phenomenon may occur, and that is the complex top of a salt body. In this part of the Sigsbee model, many areas of the top of the salt body may result in scattering of certain wavelengths resulting in short wavepath updates in FWI between scattering points (Figure 5b). So the wavepaths for the Sigsbee model though corresponding to zero offset, which often provides limited information for inversion, with double scattering it resulted in additional scattering angles (Figure 5a) and additional wavepath updates of a shorter nature. The top of the salt is a strong scatterer of energy, and thus, we may expect that such energy will be recorded. The enhanced nonlinear model update for FWI promises to offer more information, including middle wavenumbers.

We again use our 3-layer one-dimensional model in a two dimensional inversion setup using this time multi-scattered energy. Like before, we perform 15 steepest descent with line search iterations, but now including energy corresponding to fourth-order scattering (Alkhalifah and $\mathrm{Wu}, 2016 \mathrm{a})$. For the $1000 \mathrm{~m}$ maximum offset case, shown in Figure 6a, 
the inverted model in blue suffers much of the limitations of the single scattering case. The updates here are pure perturbation updates. No background wavepath updates were included as the initial model here represents the background accurately. So the difference between the spectrum of the inverted model perturbations in blue in Figure $6 \mathrm{~b}$ and the true one in red is large at the high and low ends. Having a larger maximum offset given by $3000 \mathrm{~m}$ in Figure 6c produced an inverted model given in blue that is closer to the true model given in red. The model perturbation spectrum shown in Figure 6d supports such an assertion as the middle model wavenumbers of the inverted perturbation in blue better fits the true perturbation in red. The lack of higher wavenumbers in the inverted perturbations is the result of using larger offsets as we saw earlier in the single scattering case (Figure 2d).

Compared to the single-scattering update result, we have improvements with multi scattering updates overall (or in average). However, the results of the multi scattering update are more sinusoidal, due to the admission of more higher wavenumbers over a narrow band, and this is true more for the shorter offset case. Thanks to the additional high wavenumbers, the multi scattering update results capture the sharpness of the interface better than the single scattering update result. It is important to reiterate that the multi scattering updates are not optimized, which means it is prone to the divergence of the scattering series. In the Marmousi example we will see later, we optimize the updates (fit it to the residuals, like least-squares migration). It, also, implies that the multi-scattering update results include more (possibly unwanted) variations, and thus, it is in more need for a constraint to reduce the variations.

If we add a total variation constraint to the mix, we should expect a more blocky solution, as the variations due to the sinusoidal nature of wavefields are mitigated. Figure 7a shows the inverted model in blue for the case of a $1000 \mathrm{~m}$ offset after 15 iterations. It seems to 
be similar to the single scattering result, but with slightly higher accuracy in some regions, like the velocity of the second layer up shallow. The model spectrum, shown in Figure 7b, confirms the higher agreement of the inverted perturbation in blue to the true perturbation in red. If we use offsets up to $3000 \mathrm{~m}$, the inverted model in blue in Figure 7c agrees well with the true model in red. This is further supported by the agreement in the spectrum shown in Figure $7 d$. So the additional data from the larger offsets and additional scattering managed to inject enough information into the model as it adheres to the TV constraint. The next question is how well and fast we managed to fit the data.

\section{Data fitting}

A crucial component of the experiments above is the convergence speed in which we managed to fit the data (an important goal considering we collected such data). We have restricted all the tests to fifteen iterations to allow for a fair comparison. The shot gather data residuals between the true and initial model is shown in on the left side of Figure 8. It represents the starting point of the misfit, which, as expected, reflects the need to predict model components that will reduce such reflection residuals. The data misfit for each maximum offset test $(1000 \mathrm{~m}$ and $3000 \mathrm{~m})$ is the same at the first iteration as we start from the same initial model so we normalize each offset experiment by the misfit at the first iteration, shown in Figure 8. Figure 9a shows the misfit based on the $l_{2}$ norm objective function, normalized by its value at the first iteration, as a function of iteration for the case of a maximum offset of $1000 \mathrm{~m}$ for the four experiments: conventional single scattering update in solid red, including fourth order scattering in the update in solid blue, and adding the TV constraint to both cases are the dashed counterparts. For the short offset case, and with limited middle model wavenumber information, the TV led to good fitting early, but ultimately, the low 
and middle wavenumbers were guided by the quest to reduce the variation (less constraints from the data), and the fit was worse in both the single and the multi scattered update cases. The data residuals at the last iteration for the $1000 \mathrm{~m}$ offset inversion for the four cases are shown in Figures 10a, 10b, 10c, and 10d, respectively. In all cases, the residuals are mainly reduced at the near offset as the inversion is focussed on fitting $1000 \mathrm{~m}$ offset. However, the level of misfit at the far offset is larger in the case of using the TV constraints. On the other hand, the TV constraints admitted lower misfits at the near offset, where the data misfit is contributing to the update.

For the $3000 \mathrm{~m}$ offset case, as shown in Figure 9b, the total variation constraint helped the data fitting. In fact, for the multi scattering update case and after 8 iterations, the convergence was fast. So the constraint, if accurate, acts as a bound helping to reduce the model space to those that comply with the constraint. The constraint also acts as an interpolant of wavenumbers, but like any interpolation operator, it is as good as the information we interpolate from. With multi scattering in the FWI update and large offsets, we interpolate from rich sources of data information. The data residuals at the last iteration for the $3000 \mathrm{~m}$ offset inversion for the four cases are shown in Figures 11a, 11b, 11c, and 11d, respectively, after multiplying them by a factor of 20 (left flanks of the shot gathers). The scaling of the residuals was needed to make them observable for this $3000 \mathrm{~m}$ offset case, compared to the $1000 \mathrm{~m}$ offset, which was shown at the same scale as the shot gather. Otherwise, for the $3000 \mathrm{~m}$ offset models, the residuals plotted at the same scale would have shown near zero values everywhere. So as expected, with the $3000 \mathrm{~m}$ offset inversion we obtain better fit to the data at far offsets. We also obtain the best fit (least residuals) for the case of using multi scattering updates and a TV constraint as shown in Figure 11d. 


\section{A MARMOUSI TEST}

We next test the benefits of including multi-scattering updates and the TV constraint on the Marmousi model dataset. Figure 12a shows the Marmousi model (Versteeg, 1994). We use only $3 \mathrm{~Hz}$ frequency data in this test for simplicity and since our objective is to highlight the advantages of including multi-scattering updates and the TV regularization. We use a free surface boundary condition on the top boundary. Such data were acquired assuming every point on the surface at a $25 \mathrm{~m}$ spacing as a receiver. Sources are placed at a $100 \mathrm{~m}$ spacing. We start with a smoothed version of the Marmousi shown on Figure $12 \mathrm{~b}$, as the initial model. Figure 13a shows the inversion result after 20 iterations of optimized (where the update fits the residual, Gauss Newton) single scattering updates. The inverted model, considering we use only $3 \mathrm{~Hz}$ frequency data, has some of the expected features of the true model, but also is relatively jittery (no smoothing was used here). With the same number of iterations of double scattering optimized updates, the inverted model shown in Figure 13b shows generally more of the true velocity details than its single scattering counterpart, and less harmonics as more model wavenumbers are included. Since we use the direct solver to evaluate the Helmholtz operator, the cost of using double scattering in the updates of FWI is only fractionally higher than using only single scattering. The full Hessian includes double scattering, however, not the properly weighted kind (Alkhalifah and Wu, 2016a). In other words, it does not utilize the Gauss-Newton Hessian of the second-order scattering term. Such a Gauss-Newton weighting function can be extracted mathematically from the third-order term of the generalized Taylor series expansion of the objective function with respect to the model, which we practically ignore.

When we include total variation minimization in the objective function, we expect the 
result to be more blocky. Contrary to using TV as a constraint, the minimization approach spares us the need to define the constraint value, which might be hard in complex models with low velocity zones. Figure $14 \mathrm{a}$ shows the inverted model for the $3 \mathrm{~Hz}$ data with minimizing the total variation and after 20 iterations of single scattering optimized updates. The model has a more blocky look to it. If we utilize double scattering in the update, as shown in Figure 14b, the inverted model appears to be cleaner than its single scattering counterparts (less jitters), which is courtesy of the additional model wavenumbers constrained by including double scattered energy.

\section{DISCUSSION}

The role of total variation (TV) as a constraint is interesting. In the 3-layer numerical examples, we use the true model total variation as a constraint. In practice, we can rely on the initial model for that information. Actually, the initial model in the numerical examples has the same TV as the true model. So as we constrain the total variation, the data become crucial in figuring out the accurate velocity information within such a constraint. In fact, for the low-to-middle wavenumber end of the spectrum of the model, we are more prone to errors if data are not capable of guiding the velocity within the constrained model space. So with the larger offsets, more data, we noticed that the TV constraint helped the convergence. In this case, the TV acts in interpolating the data fit into the missing model wavenumbers. On the other hand, with only short offsets, the TV constraint slightly harmed the convergence. In deciding the TV constraint, we will have to be careful when we have, or expect, low velocity zones. If velocity is only increasing or decreasing with depth, the total variation is given by the maximum and minimum velocity. In many cases, we will need to relax the constraint to allow for low velocity zones to develop. This procedure can be done at later 
stages in the iterative process of FWI as well.

Likewise, multi scattering also provides more model wavenumber information extracted from the measured data. Of course, this conclusion corresponds to updates in the perturbations. Updates in the wavepath of multi scattered energy should help the model building problem even more so for large offsets. Combining TV with multi scattering updates provides the opportunity to use a lot of the data to find the optimal model in the constrained space. The order of scattering needed depends on the strength of the multi scattered energy. In the Marmousi example we only used double scattering and the improvements were clear. If we expect in a region that interbed multiples are present, third order scattering might be useful.

In the examples we shared, the power of TV (or possibly other) regularizations in admitting middle model wavenumbers were clear. It seems to exceed those of the multi scattering updates, as mutli scattering depends on the data and the strength of such energy in the recorded data. Optimizing such multi scattering updates to fit the residuals helps enhance its role. Yet, the role of multi scattering based updates in introducing middle model wavenumbers seems to be less than that admitted through regularization. However, we have to remember that regularizations are assumptions we make on the model, and the resulting model adhering to such assumptions (including the introduced middle model wavenumbers) is as good as our regularization assumption.

The role of including multi scattering components in the FWI update process, as mentioned earlier, can potentially inject information at both the low and high end of the model spectrum. Though multi scattering is synonymous with multiples and multiple reflections, in FWI updates, our goal is not the treatment of multiples. Eventually, in all FWI, we 
thrive to build a model that reproduces all the multiples in the data. If multiples exist, the nonlinearity of the FWI optimization problem is more severe. So nonlinear multi-scattering updates can help in the convergence regardless of the background velocity, as it is a higherorder approximation of the sensitivity of the data to the model. Even though the scattering series is not necessarily a convergent series, our implementation based on optimizing the updates does not suffer from such a problem (Alkhalifah and Wu, 2016a). In other words, the series is normalized to fit the data. In the examples in this paper, the scattering order was picked haphazardly. The purpose here is mainly to emphasize the role of higher-order scattering regardless of the order.

\section{CONCLUSIONS}

In this study, we highlighted potential sources for the elusive middle model wavenumbers needed to build more of the velocity model, which is also instrumental in helping our iterative inversion methods to converge. Regularization imposes certain assumptions on the model, and in our case we tested the minimizing or constraining of the total variation (TV) of the model. As a result, the TV injected some of the necessary model wavenumbers needed to adhere to the TV assumptions. The gradient of the total variation regularization reflects the wavenumbers that will be added to the model, and those often includes middle, as well as high, wavenumbers. However, those model wavenumbers are as good as data guides them. So additional data like large offsets and multi scattered energy in the update can help the constrained FWI converge better to a better model. A single frequency experiment on the Marmousi model demonstrated how we managed to expand the wavenumber band using multi scattered energy in the FWI update and total variation regularization. 


\section{ACKNOWLEDGMENTS}

${ }_{483}$ We thank KAUST for its support. We thank the associate editor Antoine Guitton, as well

484 as reviewer Ettore Biondi, and two anonymous reviewers for their critical and helpful review 485 of the paper. 


\section{REFERENCES}

Aki, K., and P. G. Richards, 1980, Quantitative seismology - Theory and methods: W. H. Freeman \& Co.

Alkhalifah, T., 2014, Scattering-angle based filtering of the waveform inversion gradients: Geophysical Journal International, 200, 363-373.

—_ 2016a, Full-model wavenumber inversion: An emphasis on the appropriate wavenumber continuation: GEOPHYSICS, 81, R89-R98.

— $2016 \mathrm{~b}$, Research note: Insights into the data dependency on anisotropy: an inversion prospective: Geophysical Prospecting, 64, 505-513.

Alkhalifah, T., and Z. Wu, 2016a, Multiscattering inversion for low-model wavenumbers: GEOPHYSICS, 81, R417-R428.

— $2016 \mathrm{~b}$, The natural combination of full and image-based waveform inversion: Geophysical Prospecting, 64, 19-30.

Baumstein, A., 2013, Pocs-based geophysical constraints in multi-parameter full wavefield inversion: 2013 European association of geoscientistsand engineers Expanded abstract.

Bunks, C., F. Saleck, S. Zaleski, and G. Chavent, 1995, Multiscale seismic waveform inversion: GEOPHYSICS, 60, 1457-1473.

Chavent, G., and R. Plessix, 1999, An optimal true-amplitude least-squares prestack depthmigration operator: Geophysics, 64, 508-515.

Claerbout, J. F., 1985, Imaging the Earth's Interior: Blackwell Scientific Publications.

Clement, F., G. Chavent, and S. Gmez, 2001, Migration based traveltime waveform inversion of 2-d simple structures: A synthetic example: GEOPHYSICS, 66, 845-860.

Fleury, C., and F. Perrone, 2012, Bi-objective optimization for the inversion of seismic reflection data: Combined fwi and mva: SEG Technical Program Expanded Abstracts 
2012, 548, 1-6.

Guitton, A., 2012, Blocky regularization schemes for fullwaveform inversion: Geophysical Prospecting, 60, 870-884.

Jin, S., R. Madariaga, J. Virieux, and G. Lambar, 1992, Two-dimensional asymptotic iterative elastic inversion: Geophysical Journal International, 108, 575-588.

Ma, Y., D. Hale, B. Gong, and Z. Meng, 2012, Image-guided sparse-model full waveform inversion: GEOPHYSICS, 77, R189-R198.

Miller, D., M. Oristaglio, and G. Beylkin, 1987, A new slant on seismic imaging: Migration and integral geometry: GEOPHYSICS, 52, 943-964.

Mora, P., 1988, Elastic wavefield inversion of reflection and transmission data: Geophysics, 53, 750-759.

Portniaguine, O., and M. S. Zhdanov, 1999, Focusing geophysical inversion images: Geophysics, 64, 874-887.

Pratt, R. G., Z.-M. Song, P. Williamson, and M. Warner, 1996, Two-dimensional velocity models from wide-angle seismic data by wavefield inversion: Geophysical Journal International, 124, 323-340.

Press, W. H., B. P. Flannery, S. A. Teukolsky, and W. T. Vetterling, 1986, Numerical recipes: Cambridge University Press.

Sirgue, L., and G. Pratt, 2004, Efficient waveform inversion and imaging: a strategy for selecting temporal frequencies: Geophysics, 69, 231-248.

Tarantola, A., 1984, Inversion of seismic reflection data in the acoustic approximation: Geophysics, 49, 1259-1266.

Thierry, P., S. Operto, and G. Lambare, 1999, Fast 2-d ray+born migration/inversion in complex media: GEOPHYSICS, 64, 162-181. 
Versteeg, R., 1994, The marmousi experience: Velocity model determination on a synthetic complex data set: The Leading Edge, 13, 927-936.

Virieux, J., and S. Operto, 2009, An overview of full-waveform inversion in exploration geophysics: GEOPHYSICS, 74, WCC1-WCC26.

Wang, S., F. Chen, H. Zhang, and Y. Shen, 2013, Reflection-based full waveform inversion (rfwi) in the frequency domain: SEG Technical Program Expanded Abstracts 2013, 171, $877-881$.

Williamson, P. R., 1991, A guide to the limits of resolution imposed by scattering in ray tomography: GEOPHYSICS, 56, 202-207.

Wu, R., and N. Toksaz, 1987, Diffraction tomography and multisource holography applied to seismic imaging: GEOPHYSICS, 52, 11-25.

Xu, S., D. Wang, F. Chen, G. Lambare, and Y. Zhang, 2012, Inversion on reflected seismic wave: SEG Technical Program Expanded Abstracts 2012, 509, 1-7.

Yilmaz, O., 2001, Seismic Data Analysis: Soc. of Expl. Geophys.

Zhu, M., and T. Chan, 2008, An efficient primal-dual hybrid gradient algorithm for total variation image restoration: CAM Reports, 8-34. 


\section{LIST OF FIGURES}

1 A schematic diagram showing the potential recovered model wavenumbers at a point in the model space as a function of frequency in the data (in this case, the offset-todepth ratio seems to be small resulting in a large middle wavenumber gap). The addition of multi scattered energy with a total variation constraint has the potential to expand the yellow region of expected model wavenumbers.

2 The inverted velocity using conventional FWI and its spectrum as a function of normalized vertical wavenumbers for a maximum offset of $1000 \mathrm{~m}(\mathrm{a}, \mathrm{b})$, and $3000 \mathrm{~m}(\mathrm{c}, \mathrm{d})$. Improvements due to larger offsets appear at the bottom of the first and second layers and in the third layer. In the wavenumber spectrum, we obtain a better fit in the low end of the spectrum for larger offsets, but deteriorating results at the high end.

3 A shot gather with the left flank corresponding to three-layer model shown in Figure $2 \mathrm{a}$ in blue (the true model), and the right flank corresponding to the smoothed version of the true model shown in Figure 2a in black (the initial model).

4 The inverted velocity using conventional FWI plus TV constraint and its spectrum for a maximum offset of $1000 \mathrm{~m}(\mathrm{a}, \mathrm{b})$, and $3000 \mathrm{~m}(\mathrm{c}, \mathrm{d})$.

5 A schematic plot of scattering from the top of the Sigsbee model, for a zero offset source-receiver configuration, showing a) the potential additional scattering angles and b) the additional shorter wavepaths, attributed to double scattering.

6 The inverted velocity using mutiscattering FWI and its spectrum for a maximum offset of $1000 \mathrm{~m}(\mathrm{a}, \mathrm{b})$, and $3000 \mathrm{~m}(\mathrm{c}, \mathrm{d})$.

$7 \quad$ The inverted velocity using multi scattering FWI plus TV constraint and its spectrum for a maximum offset of 1000m (a, b), and 3000m ( c, d).

8 The left flank of the shot gather corresponds to the true velocity model, while the 
right flank corresponds to the data difference (residual) between the true and the initial velocity model, in which the starting point of the objective function in Figure 9a for offsets up to $1000 \mathrm{~m}$ and Figure $9 \mathrm{~b}$ for offsets up to $3000 \mathrm{~m}$ is calculated.

9 The misfit values based on the $l_{2}$ norm as a function of FWI iterations for a maximum offset of a) $1000 \mathrm{~m}$, and b) $3000 \mathrm{~m}$.

10 The left flanks of the shot gathers correspond to the true velocity model, while the right flanks correspond to the data difference (residual) between the true and inverted models. Thus, the residuals correspond to the end points of the objective function in Figure 9a for offsets up to $1000 \mathrm{~m}$ for (a) the conventional FWI, (b) with TV, (c) the multi scattering update, and (d) with TV for the multi scattering update.

11 The left flanks of the shot gathers correspond to the true velocity model, while the right flanks correspond to the data difference (residual) between the true and inverted models scaled up by a factor of 20 . Thus, the residuals correspond to the end points of the objective function in Figure 9b for offsets up to 3000m using (a) conventional FWI, (b) with TV, (c) the multi scattering update, and (d) with TV for the multi scattering update.

12 a) The true velocity model. b) The initial velocity model.

13 a) The inverted model after 20 iterations of optimized single scattering updates. b) The inverted model after 20 iterations of optimized double scattering updates.

14 a) The inverted model after 20 iterations of optimized single scattering updates with TV regularization. b) The inverted model after 20 iterations of optimized double scattering updates with TV regularization. 


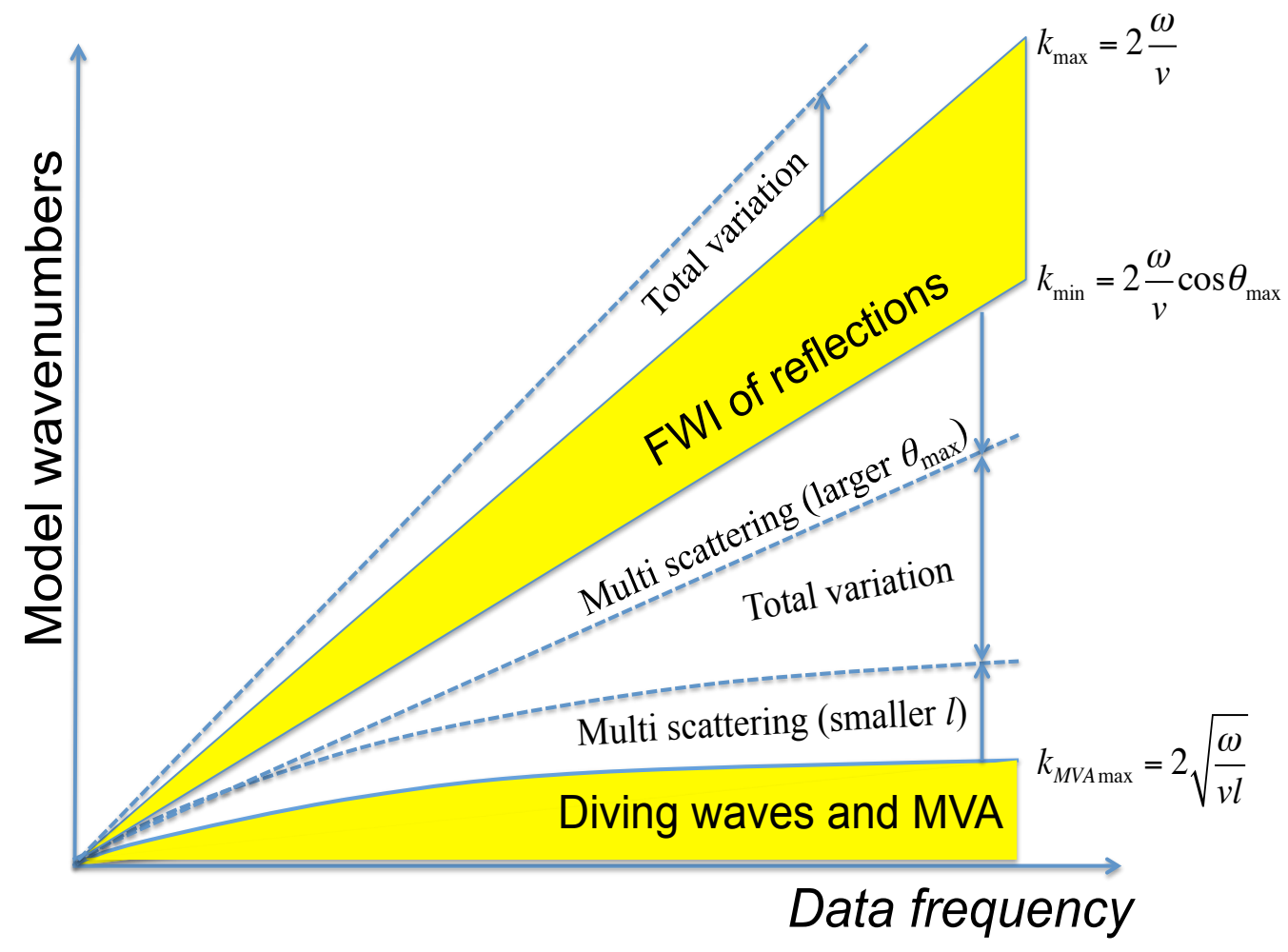

Figure 1: A schematic diagram showing the potential recovered model wavenumbers at a point in the model space as a function of frequency in the data (in this case, the offset-todepth ratio seems to be small resulting in a large middle wavenumber gap). The addition of multi scattered energy with a total variation constraint has the potential to expand the yellow region of expected model wavenumbers.

Alkhalifah - 


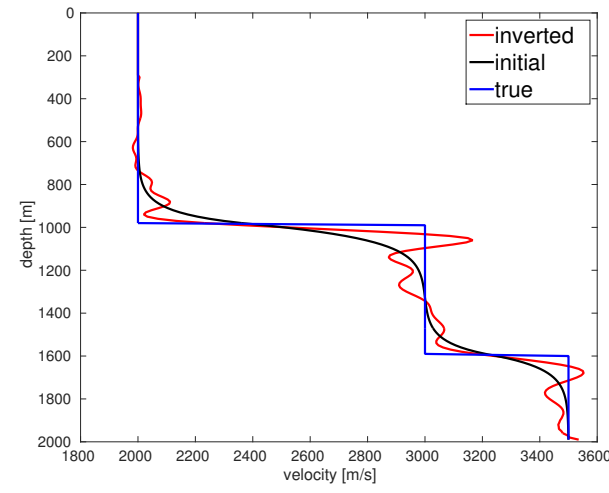

(a)

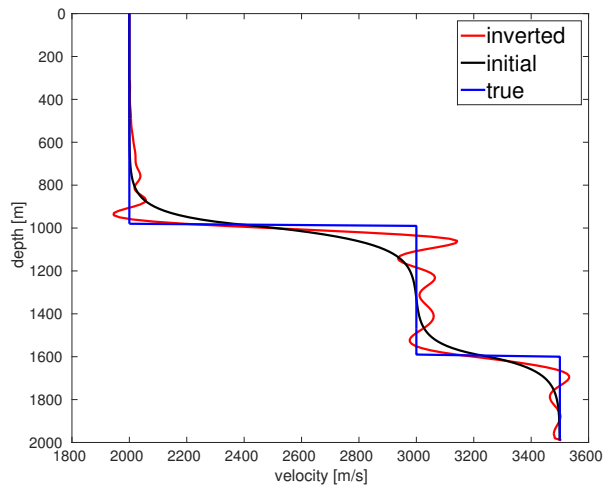

(c)

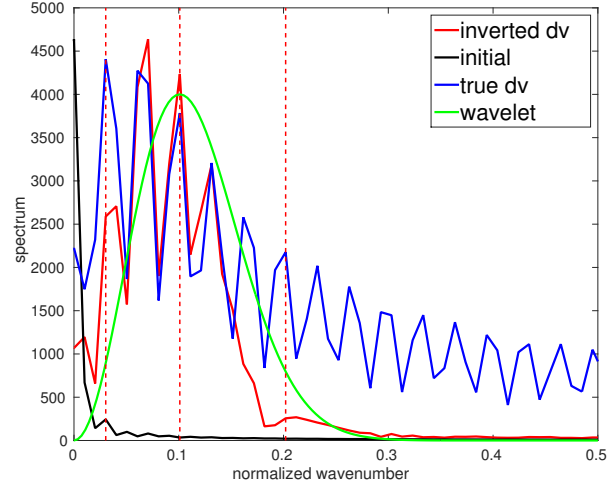

(b)

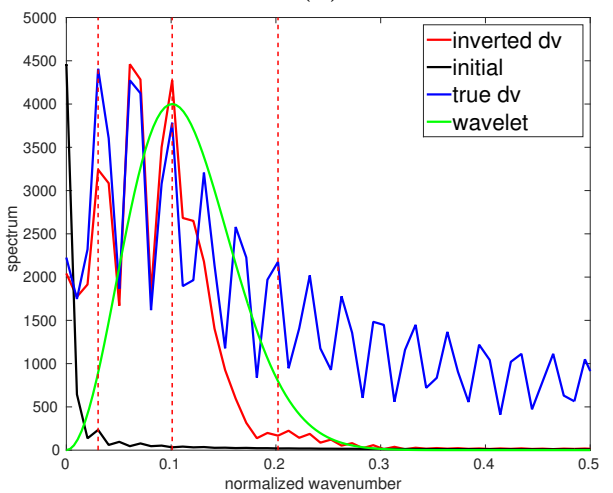

(d)

Figure 2: The inverted velocity using conventional FWI and its spectrum as a function of normalized vertical wavenumbers for a maximum offset of $1000 \mathrm{~m}(\mathrm{a}, \mathrm{b})$, and $3000 \mathrm{~m}(\mathrm{c}, \mathrm{d})$. Improvements due to larger offsets appear at the bottom of the first and second layers and in the third layer. In the wavenumber spectrum, we obtain a better fit in the low end of the spectrum for larger offsets, but deteriorating results at the high end.

Alkhalifah - 


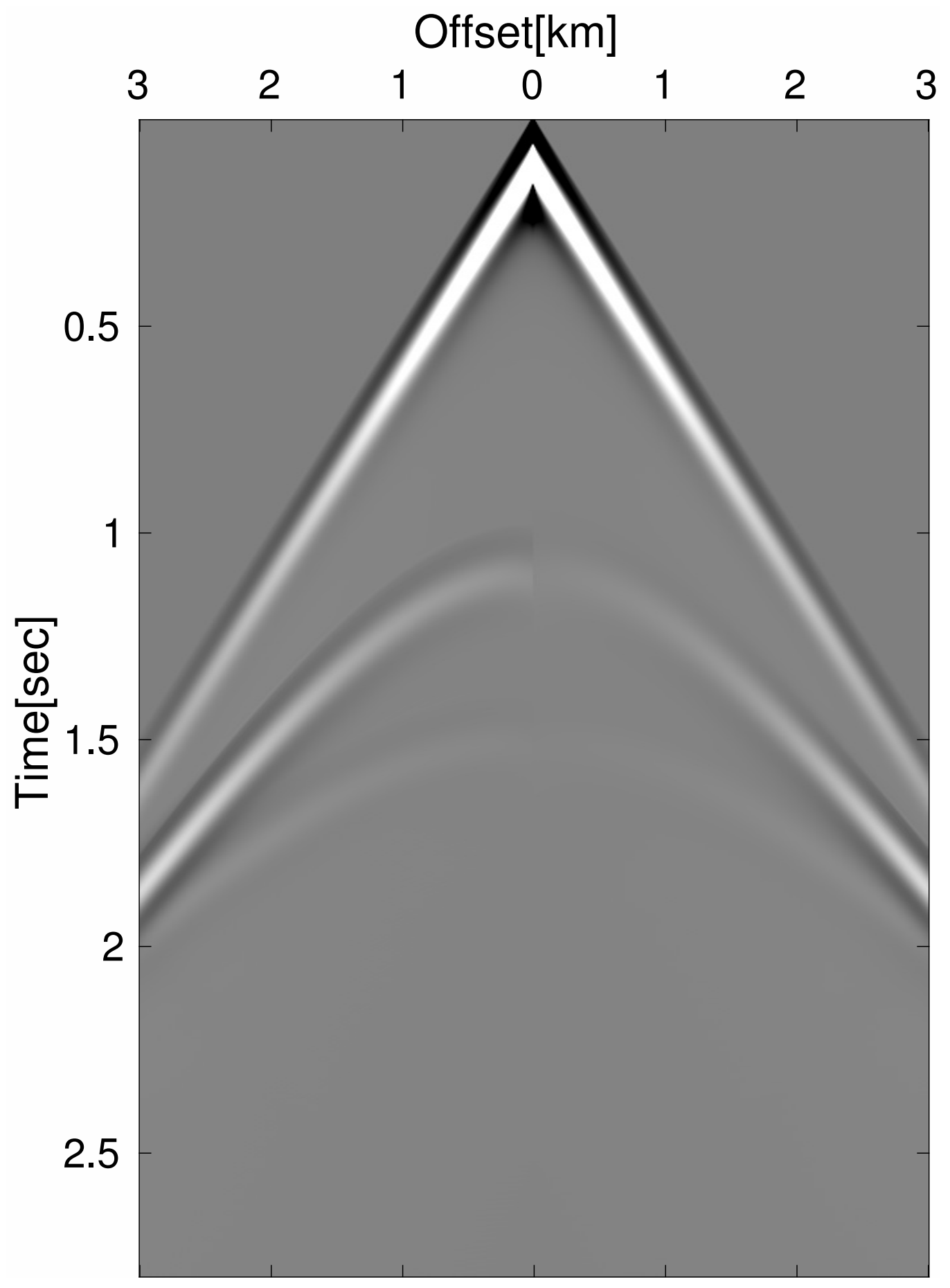

Figure 3: A shot gather with the left flank corresponding to three-layer model shown in Figure $2 \mathrm{a}$ in blue (the true model), and the right flank corresponding to the smoothed version of the true model shown in Figure 2a in black (the initial model).

Alkhalifah - 


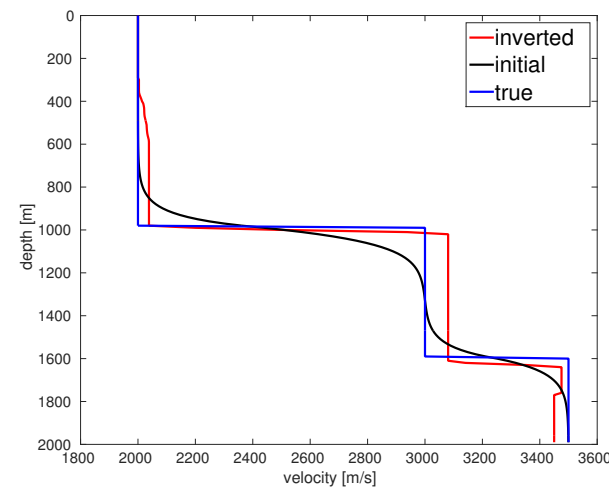

(a)

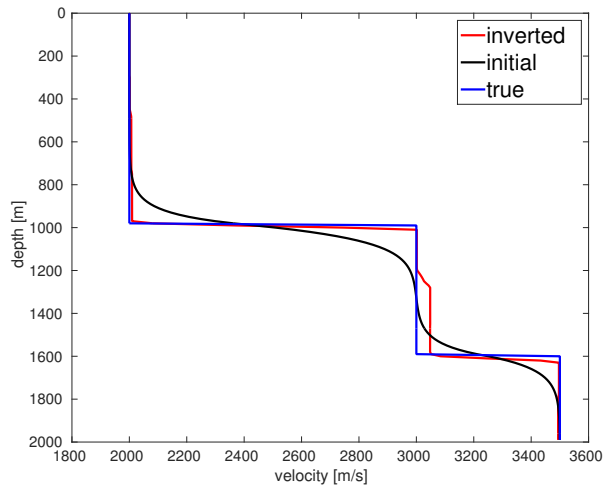

(c)

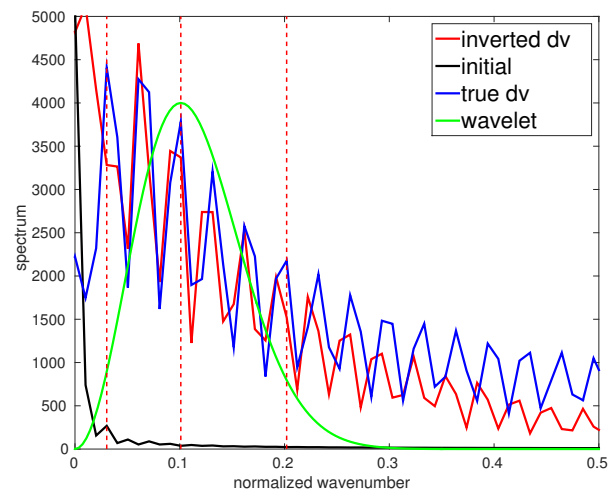

(b)

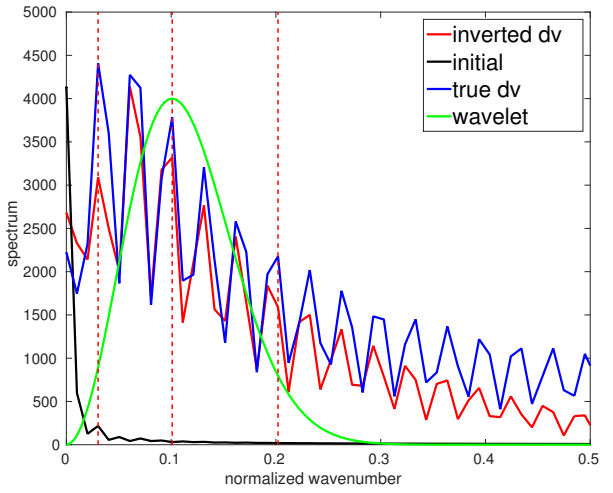

(d)

Figure 4: The inverted velocity using conventional FWI plus TV constraint and its spectrum for a maximum offset of $1000 \mathrm{~m}(\mathrm{a}, \mathrm{b})$, and $3000 \mathrm{~m}(\mathrm{c}, \mathrm{d})$.

Alkhalifah - 


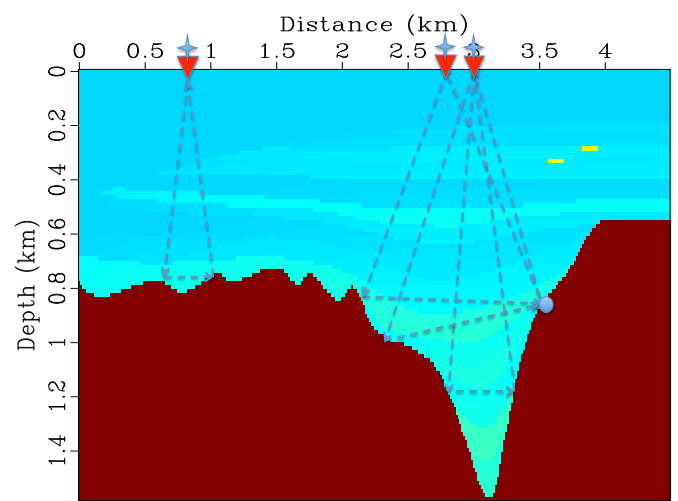

(a)

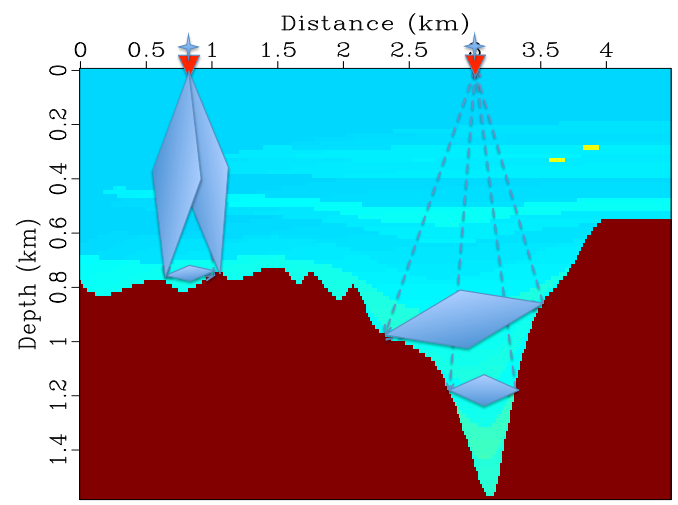

(b)

Figure 5: A schematic plot of scattering from the top of the Sigsbee model, for a zero offset source-receiver configuration, showing a) the potential additional scattering angles and b) the additional shorter wavepaths, attributed to double scattering.

Alkhalifah - 


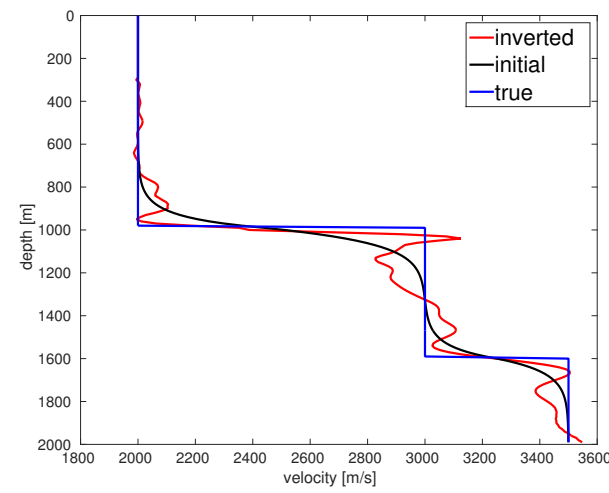

(a)

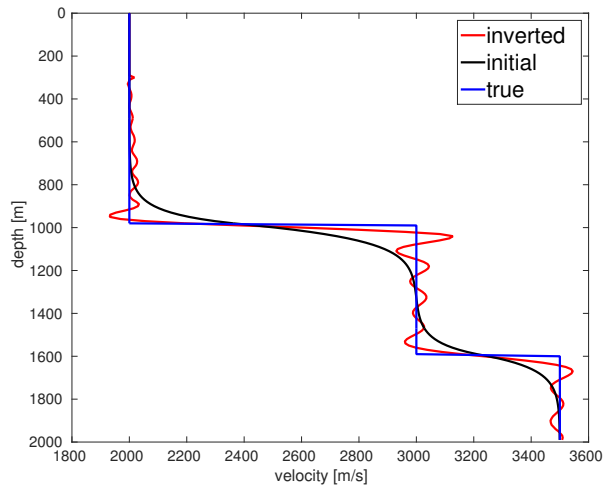

(c)

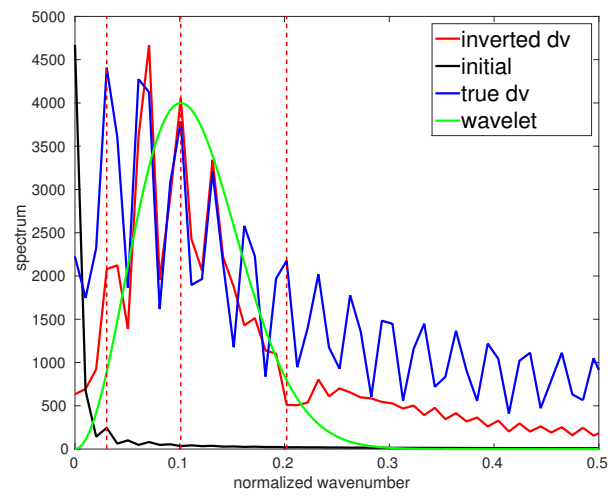

(b)

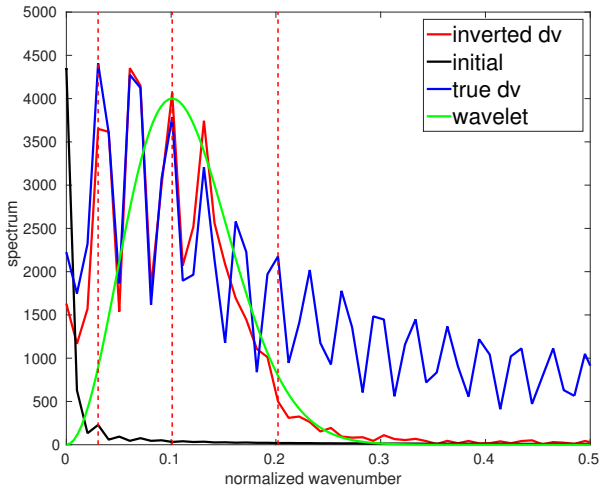

(d)

Figure 6: The inverted velocity using mutiscattering FWI and its spectrum for a maximum offset of $1000 \mathrm{~m}(\mathrm{a}, \mathrm{b})$, and $3000 \mathrm{~m}(\mathrm{c}, \mathrm{d})$.

Alkhalifah - 


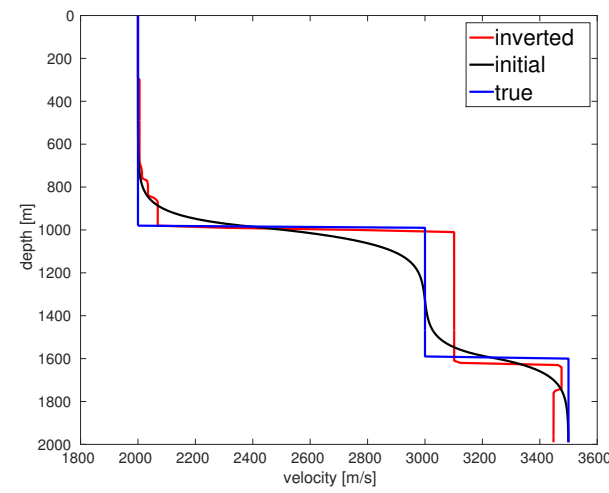

(a)

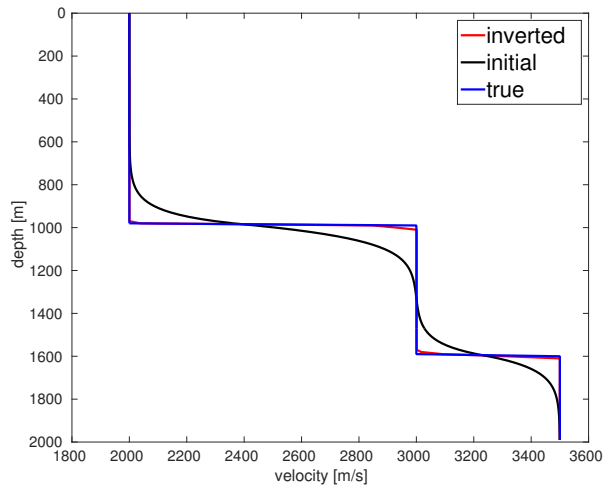

(c)

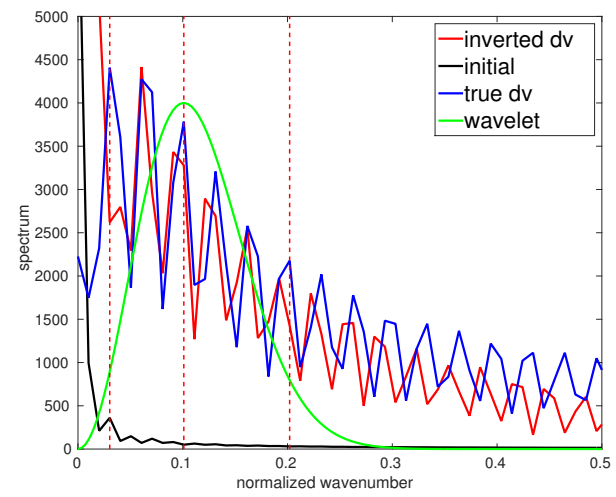

(b)

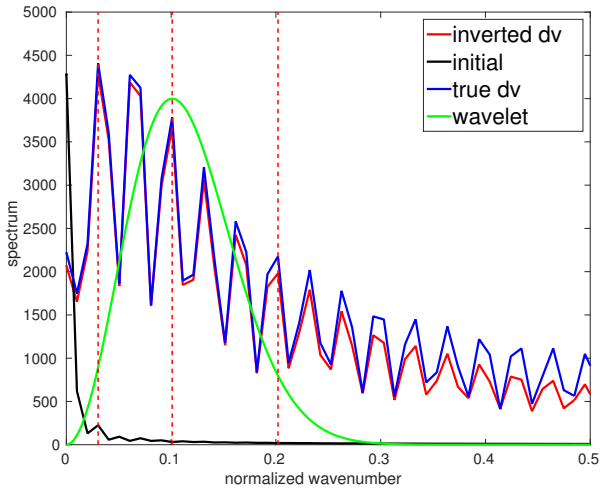

(d)

Figure 7: The inverted velocity using multi scattering FWI plus TV constraint and its spectrum for a maximum offset of $1000 \mathrm{~m}$ (a, b), and 3000m ( c, d).

Alkhalifah - 


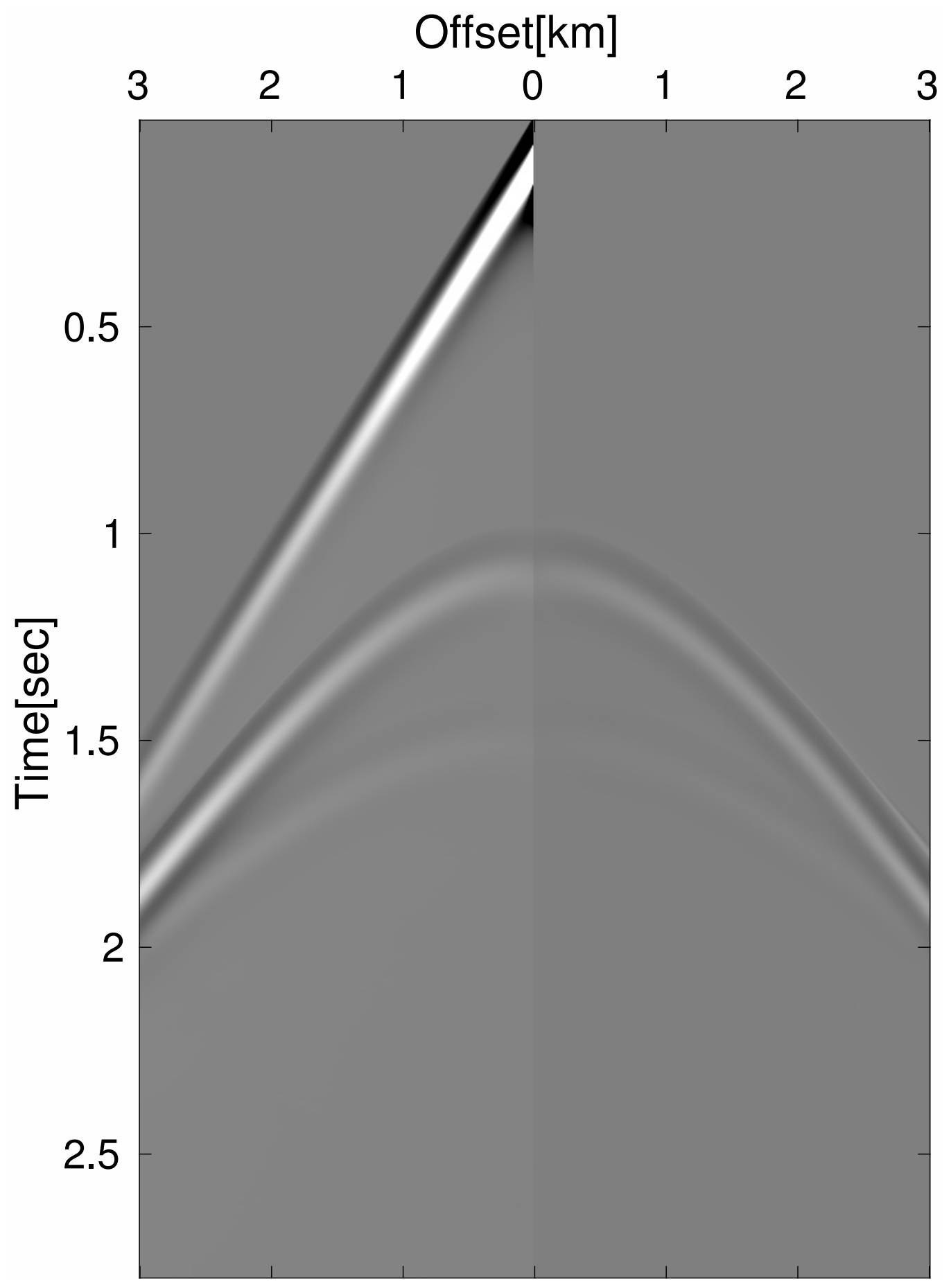

Figure 8: The left flank of the shot gather corresponds to the true velocity model, while the right flank corresponds to the data difference (residual) between the true and the initial velocity model, in which the starting point of the objective function in Figure 9a for offsets up to $1000 \mathrm{~m}$ and Figure $9 \mathrm{~b}$ for offsets up to $3000 \mathrm{~m}$ is calculated.

Alkhalifah - 


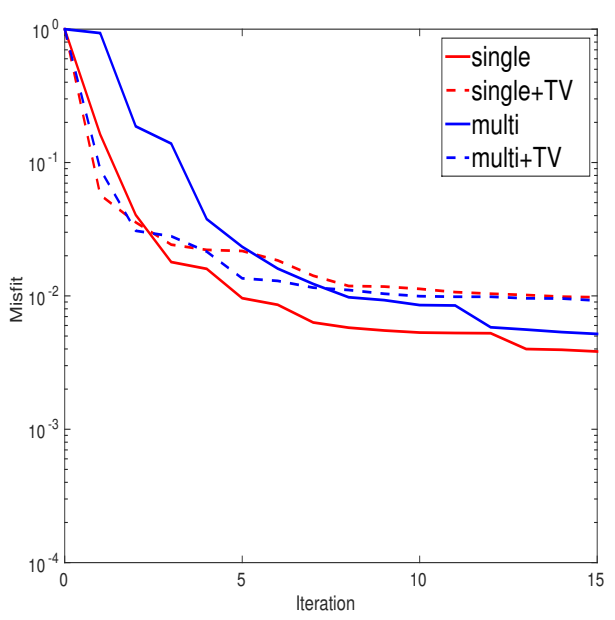

(a)

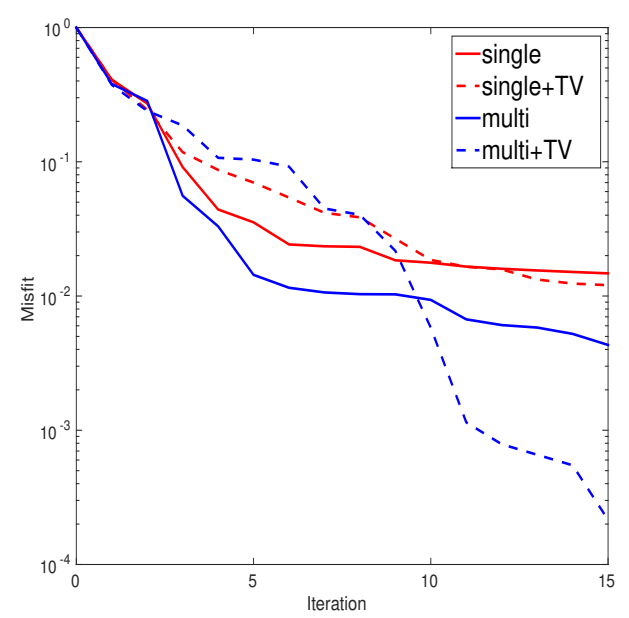

(b)

Figure 9: The misfit values based on the $l_{2}$ norm as a function of FWI iterations for a maximum offset of a) $1000 \mathrm{~m}$, and b) $3000 \mathrm{~m}$.

Alkhalifah - 


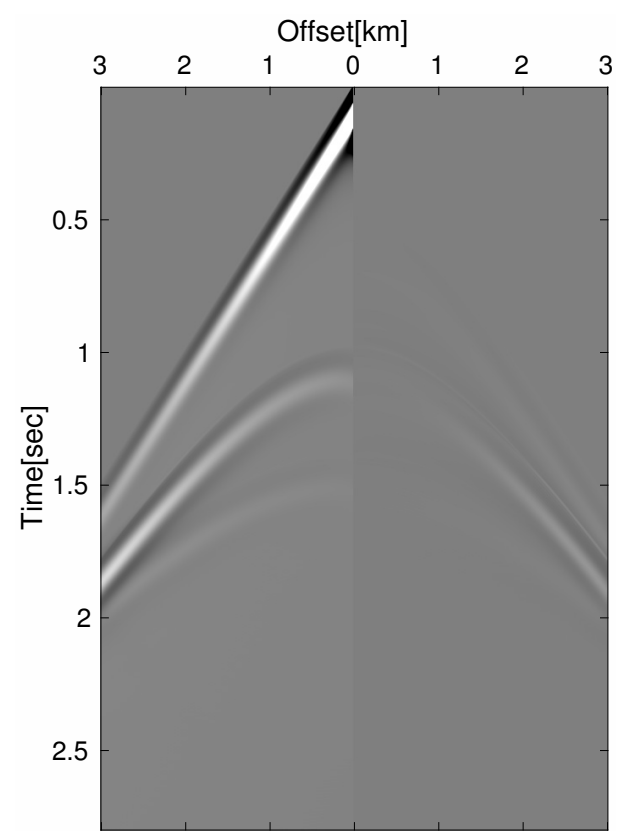

(a)

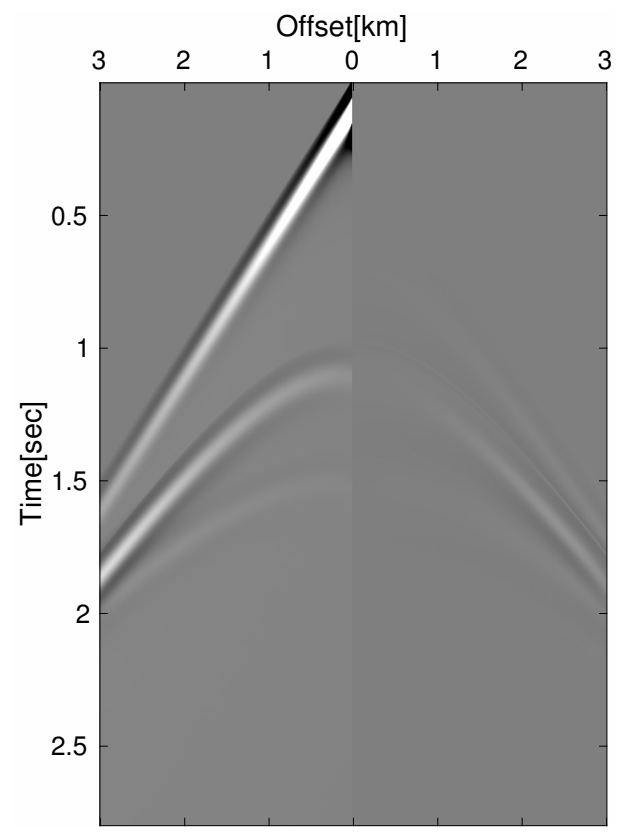

(c)

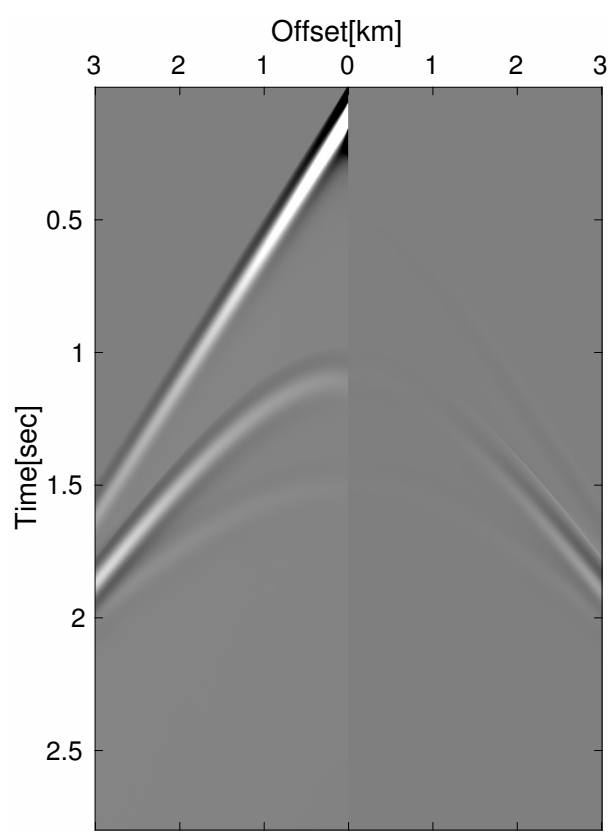

(b)

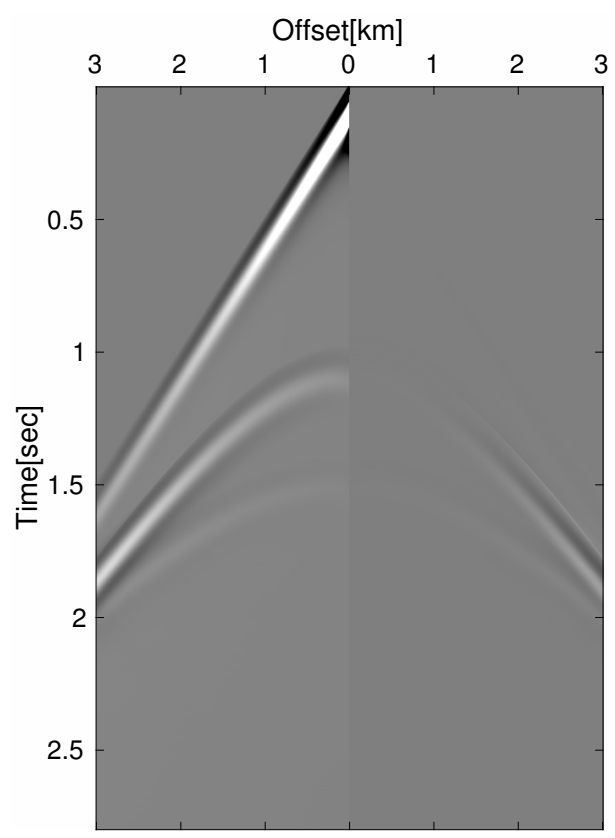

(d)

Figure 10: The left flanks of the shot gathers correspond to the true velocity model, while the right flanks correspond to the data difference (residual) between the true and inverted models. Thus, the residuals correspond to the end points of the objective function in Figure 9a for offsets up to $1000 \mathrm{~m}$ for (a) the conventional FWI, (b) with TV, (c) the multi scattering update, and (d) with TV for the multi scattering update. 


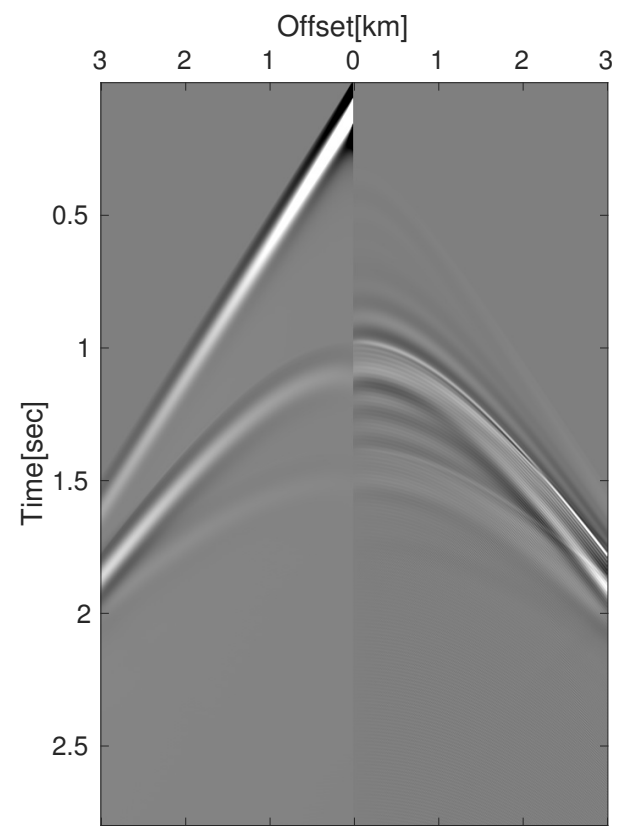

(a)

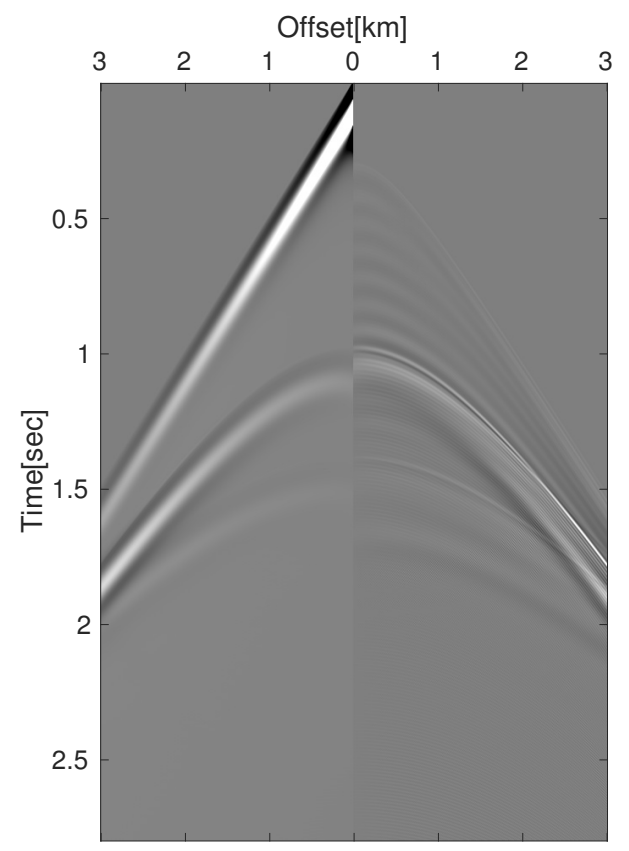

(c)

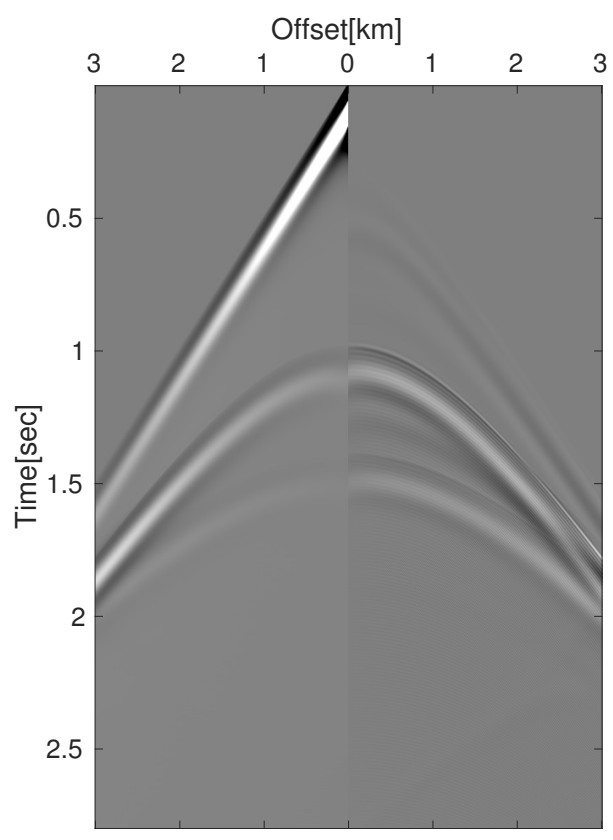

(b)

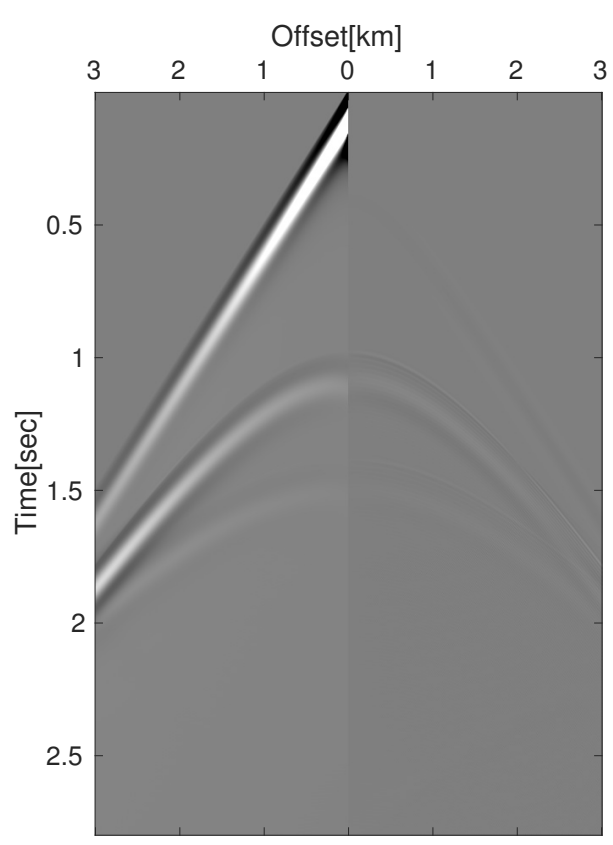

(d)

Figure 11: The left flanks of the shot gathers correspond to the true velocity model, while the right flanks correspond to the data difference (residual) between the true and inverted models scaled up by a factor of 20 . Thus, the residuals correspond to the end points of the objective function in Figure $9 \mathrm{~b}$ for offsets up to 3000m using (a) conventional FWI, (b) with TV, (c) the multi scattering update, and (d) with TV for the multi scattering update. 


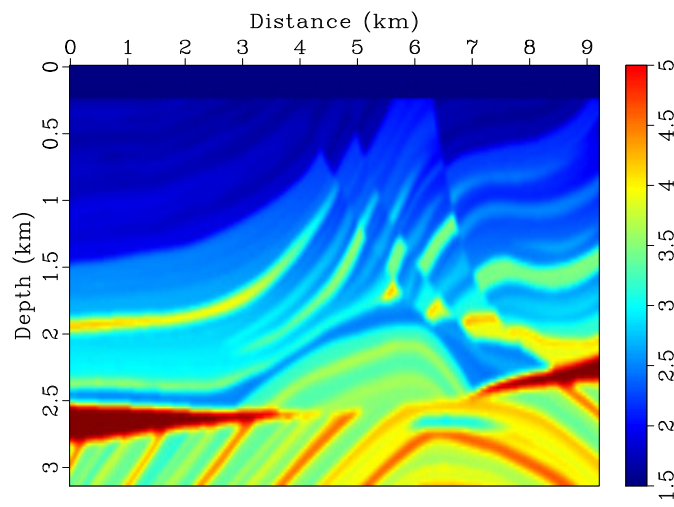

(a)

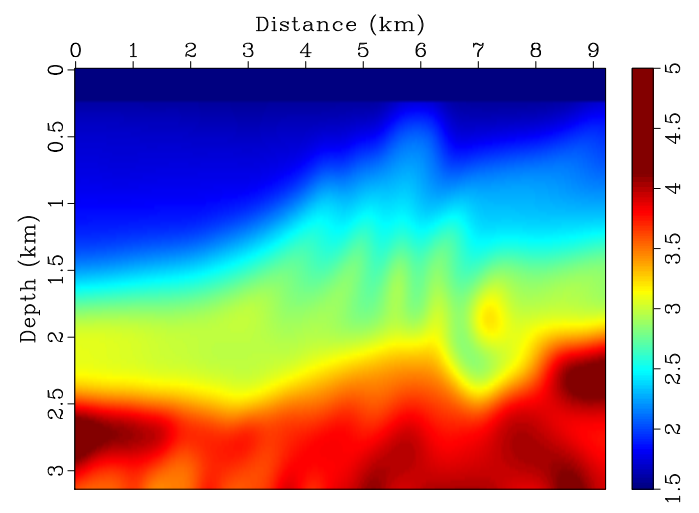

(b)

Figure 12: a) The true velocity model. b) The initial velocity model. Alkhalifah - 


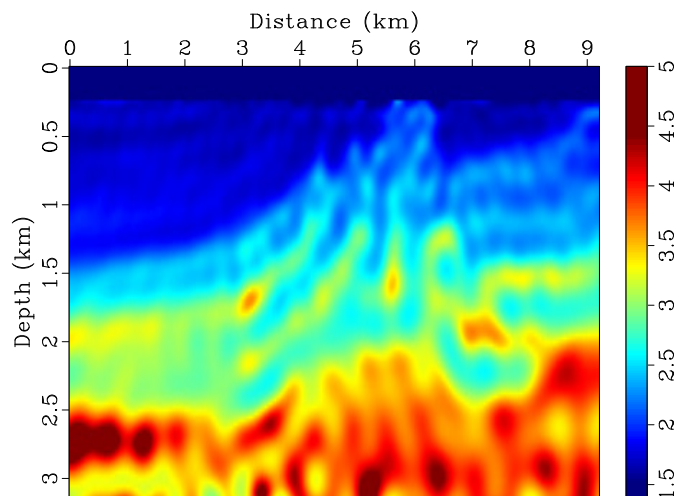

(a)

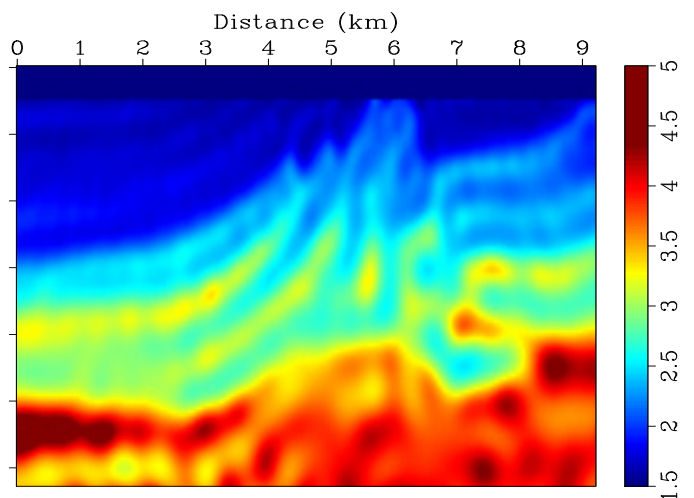

(b)

Figure 13: a) The inverted model after 20 iterations of optimized single scattering updates. b) The inverted model after 20 iterations of optimized double scattering updates. Alkhalifah - 


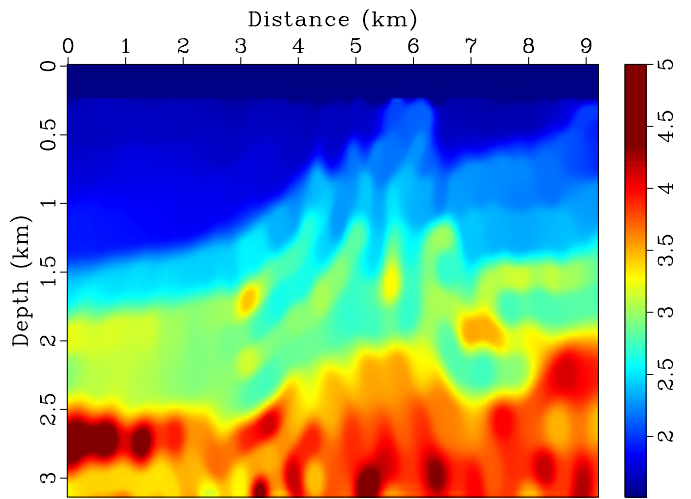

(a)

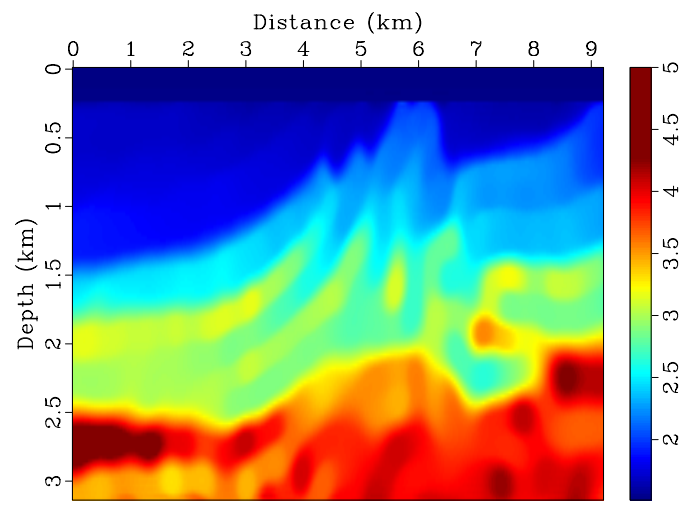

(b)

Figure 14: a) The inverted model after 20 iterations of optimized single scattering updates with TV regularization. b) The inverted model after 20 iterations of optimized double scattering updates with TV regularization.

Alkhalifah - 